\title{
Relación entre periodo de amamantamiento y la presencia de hábitos nocivos en niños Venezolanos
}

\section{Resumen}

Objetivo: Establecer la relación entre período de amamantamiento y la presencia de hábitos nocivos en niños entre 3 y 6 años. Materiales y métodos: Estudio transversal y observacional, con una muestra de 37 niños que acudieron a la consulta de niño sano en el Ambulatorio Docente del Hospital Universitario de Caracas durante el mes de agosto del año 2013. Bajo aprobación y firma del consentimiento informado se entrevistaron a las madres para determinar su nivel educativo, la duración del período de amamantamiento y presencia hábitos viciosos bucales como succión de dedo u objeto, alergias y problemas de otorrinolaringología, un examen clínico bucal para el análisis de hábitos de de-
Blanca Dickmar Lozada D. ${ }^{1}$

María de las Nieves Hernández $z^{2}$

Maria Elena Guerra G. ${ }^{3}$

Artigo Original

\section{Relação entre período de aleitamento ea presença de hábitos nocivos em crianças Venezuelanas}

\section{Resumo}

Objetivo: estabelecer a relação entre período de aleitamento ea presença de hábitos nocivos em crianças entre 3 e 6 anos. Materiais e métodos: Es- glución atípica y respiración bucal. Resultados: Se observó que aquellos pacientes que fueron amamantados por un periodo mayor a 6 meses presentaron menos enfermedades alérgicas y de Otorrrinolaringología, así como menos presencia de hábitos viciosos bucales como: uso de chupete, biberón, deglución atípica y respiración bucal. Conclusión: Se observa en los niños estudiados la importancia del amamantamiento en los primeros meses de vida para lograr una maduración adecuada en las funciones del aparato bucal, sin presencia de hábitos viciosos bucales, así como también un crecimiento y desarrollo óptimo de su estructura cráneo facial.

Palabras Clave: Amamantamiento, hábitos viciosos bucales, niños.

${ }^{1}$ Residente del Postgrado de Odontología Infantil Facultad de Odontología Universidad Central de Venezuela

${ }^{2}$ Especialista en Odontología Infantil. Profesora Asociada. Centro de Investigación y Atención a pacientes con malformaciones Cráneo-Faciales y Prótesis Maxilo - Facial. Facultad de Odontología Universidad Central de Venezuela

${ }^{3}$ Dra. En Ciencias Mención Salud Bucal Materno Infantil. Profesora Titular. Centro de Atención a Pacientes con Enfermedades Infectocontagiosas Dra. Elsa La Corte. Facultad de Odontología Universidad Central de Venezuela 
as mães para determinar seu nível de educação, a duração do aleitamento materno e a presença hábitos nocivos, como sucção de dedo ou objeto, alergias e problemas otorrinolaringológicos,e se realizou um exame oral para a análise dos hábitos bucais nocivos como uso de chupeta, mamadeira, deglutição atípica e respiração bucal. Resultados: Observou-se que os pacientes que foram amamentadas por mais de 6 meses e tinham menos doenças alérgicas e de Otorrrinolaringologia, bem como menor presença hábitos orais nocivos, tais como o uso de chupeta, mamadeira, deglutição atípica e respiração bucal. Conclusão: Foi observada nas crianças estudadas a importância da amamentação nos primeiros meses de vida para alcançar a maturação adequada das funções bucais, sem a presença de hábitos orais nocivos, bem como o crescimento e o desenvolvimento adequados da estrutura craniofacial.

Palavras-chave: Aleitamento materno, boca hábitos viciosos, crianças.

\section{Original article}

\section{Relationship between breast-feeding and the presence of harmful habits in Veneuelan children}

\begin{abstract}
Objetive: To establish the relationship between breast-feeding and the presence of harmful habits in children between 3 and 6 years. Materials and methods: A cross-sectional study and observational, with a sample of 37 children who attended the consultation of healthy child in the Outpatient Clinic Teaching Hospital of the University of Caracas during the month of August 2013. Upon approval and signing of the informed consent is interviewed the mothers to determine their level of education, the duration of the suckling period and vicious habits presence as oral suction of finger or object, allergies, and problems in otolaryngology, a clinical examination to the oral analysis of habits of atypical deglutition and mouth breathing. Results: It was observed that patients who were breastfed for a period greater than 6 months were less allergic diseases and Otorrrinolaringologia, as well as less of a presence of vicious oral habits such as: use of a pacifier, bottle, atypical deglutition
\end{abstract}

and mouth breathing. Conclusion: We observed in the children studied the importance of breastfeeding in the first few months of life to achieve a proper maturation in the functions of the mouthparts, without the presence of oral vicious habits, as well as a growth and optimum development of their facial skull structure.

Key Words: breastfeeding, oral vicious habits children.

\section{Introducción}

El amamantamiento es la forma ideal de alimentación del recién nacido, ya que a través de la función instintiva de succión del seno materno proporciona que la leche se dirija directamente a la boca promoviendo el acto reflejo de la deglución, luego el alimento va al esófago y posteriormente al estómago. De esa forma, la práctica del amamantamiento natural desempeña un importante papel en la salud del ser humano, además de actuar directamente en la nutrición, también 
trae el refuerzo del sistema inmunológico y psicológico del niño ${ }^{1 ; 2}$.

El amamantamiento madura las funciones del aparato bucal, para el buen desarrollo y crecimiento normal dento-buco-maxilo-facial, por proporcionar la acción adecuada de los músculos peri-orales principalmente los labios e intraorales los de la lengua, toda esta biomecánica de succión es diferente cuando se emplea un biberón, el cual va a involucrar una incorrecta postura de los músculos intra-bucales especialmente la lengua, la cual se ubica en en el espacio oral funcional que no le corresponde, señalada en la etiología de la presencia de hábitos viciosos bucales y asociada al retraso en el crecimiento óseo, malas posiciones dentales, disturbios en la succión y deglución así como también de respiración, dificultad en el habla y perturbación en el equilibrio de la musculatura dento-bucomaxilo-facial ${ }^{1 ; 3}$.

Los hábitos nocivos bucales se definen como la costumbre que se adquiere de un acto por su repetición frecuente. No son funcionales ni necesarios y povocan fuerzas no naturales sobre los dientes que pueden causar alteraciones dentobuco-maxilo-faciales ${ }^{4}$; aparecen entre el 56\% y $75 \%$ de los niños; producen alteraciones del balance neuromuscular, discrepancia entre hueso y diente, mordida cruzada posterior y anterior, mordida abierta anterior y pueden ocasionar desviaciones de la mandíbula ${ }^{5}$. Muy pocas anomalías buco-dento-maxilo-faciales se pueden prevenir, pero al interceptarlas en infantes se estarían eliminando factores etiológicos y mejorar en un $25 \%$ de los pacientes ${ }^{6}$.

La importancia del estudio entre la relación del periodo de amamantamiento exclusivo en la prevención del establecimiento de hábitos nocivos bucales y el desarrollo de anomalías dento- buco-maxilo-faciales nos motivó para realizar este estudio.

\section{Objetivo}

Correlacionar el período de amamantamiento con la presencia de los hábitos nocivos bucales en los niños que acuden a la consulta pediátrica de niño sano al Ambulatorio Docente Hospital Universitario de Caracas julio-agosto 2013.

\section{Materiales y métodos}

Estudio de campo, no experimental, con el propósito correlacionar las variables y analizar su incidencia e interrelación en un momento dado. Analítico de corte transversal, cuyo método se basó en un cuestionario a las madres y la observación bucal de los niños.

El tamaño de la muestra fue a convenir, se seleccionaron las madres que aceptaron participar en el estudio y firmaron el consentimiento informado, estas eran incorporadas al estudio sí niños permitieron la evaluación bucal espontáneamente.

Para la recolección de datos se utilizó un instrumento adaptado de la historia clínica de la Cátedra de Odontología Pediátrica de la Universidad Central de Venezuela, que fue sometido y ajustado para este estudio, a las madres se aplicó un cuestionario para determinar variables sociodemográficas, el tiempo de amamantamiento, comienzo de alimentación con biberón, historia y consultas previas por enfermedades alérgicas y de otorrinolaringología, así como señalar la presencia de hábitos nocivos de masticación de objetos y succión de dedo, objeto o pañal ya que se podían pasar por alto al momento del examen. A todos los niños se 
les realizó una evaluación bucal, para recolectar componentes clínicos relacionados con la presencia de hábitos nocivos.

El examen clínico, fue realizado por una estudiante del postgrado de odontología infantil de la Facultad de Odontología de la Universidad Central de Venezuela, previo entrenamiento. Obteniendo un Kappa intraexaminador de 0,77 (IC 0,70-0,83).

La observación clínica de hábitos viciosos bucales y medición la realizó por medio un examen bucal, con el niño sentado en una silla de la sala de espera. La examinadora con guantes, un fronto-luz y dos paletas de madera, evaluaba, la relación molar, y la relación dental anterior, para la determinación clínica de los hábitos de succión y deglución se le indicaba al niño que tragara saliva y se observaba el movimiento de los músculos peribucales y se palpaban los temporales. Para la evaluación de respiración bucal se tomaron las características faciales, labio superior corto, cara alargada, visibilidad dentaria, se le indicaba al niño que pegará sus labios al cerrar la boca y se observaba la capacidad de mantener los labios cerrados y respirar. Se observaron las manos para ver hábito de onicofagia.
Aspectos éticos del estudio: Para la recolección se informó a la madre de los niño/as, explicándoles los objetivos de la investigación y bajo su consentimiento informado aprobado y avalado por la Comisión de Bioética de la Facultad de Odontología bajo Nº466-2013. Aquellos niños cuyas madres accedieron voluntariamente y firmaron el consentimiento, participaron y fueron incluidos en el estudio.

Los datos recolectados fueron introducidos en la base de datos del programa estadístico "Statistical Package for the Social Sciences" (SPSS) versión 20.0. Todos los análisis se efectuaron con un intervalo de confianza del 95\%. Tomamdo la correlación de Pearson para determinar la significancia estadística.

\section{Resultados}

\section{Analisis descriptivo:}

Todas las madres reportaron no haber concluido el bachillerato.

Del total de la muestra estudiada, el 40,54\% (15) pertenecen al género femenino y 59,46\% (22) al género masculino.

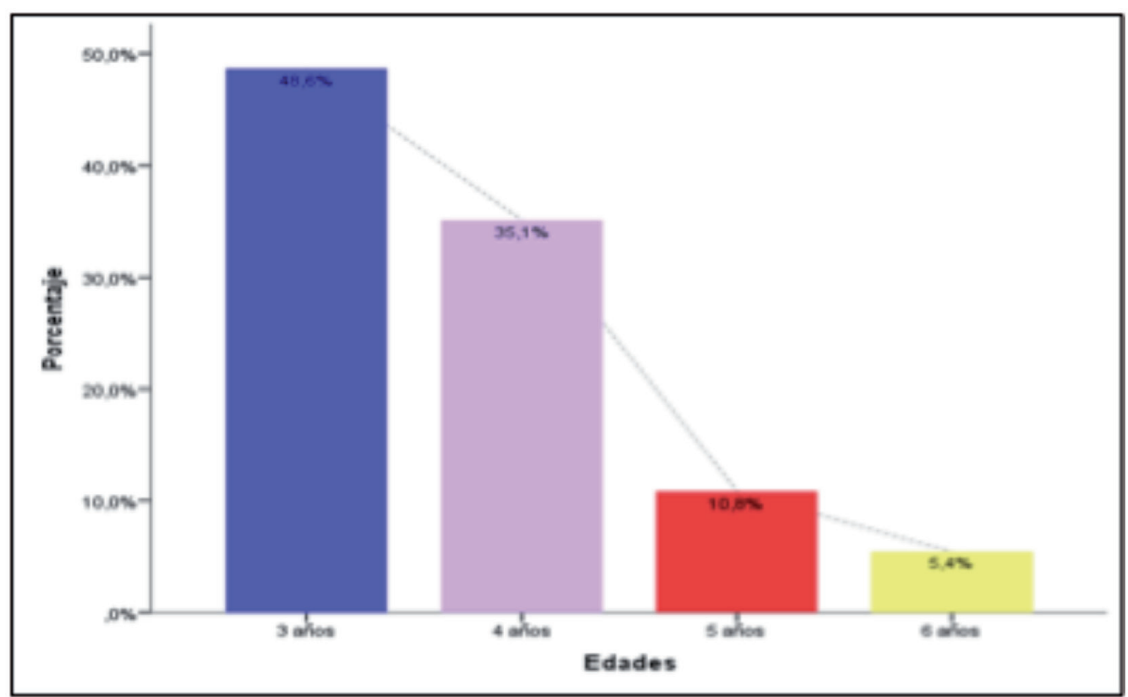

Figura 1. Porcentaje de individuos por edades. 


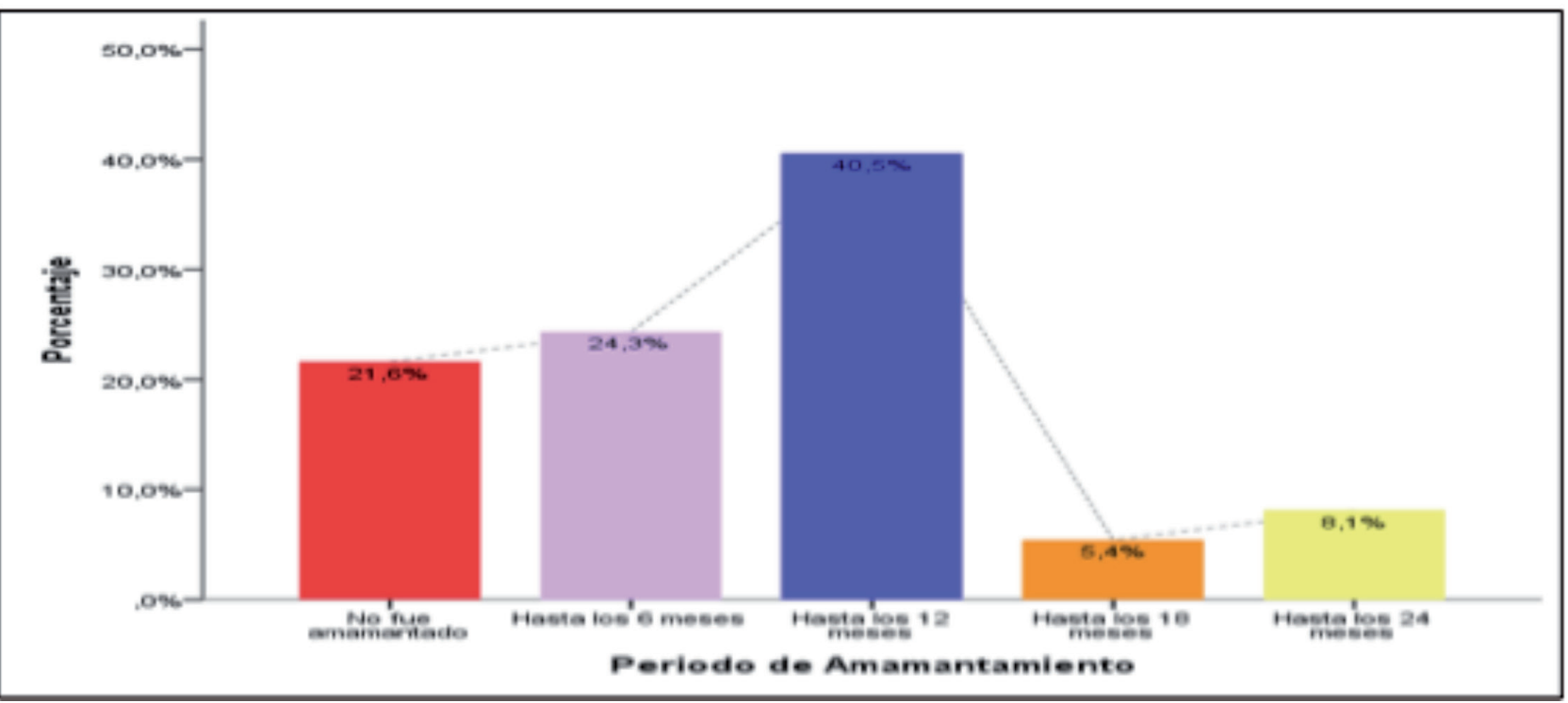

Figura 2. Porcentaje de individuos según el periodo de amamantamiento.

El 48,6\% (18) tenían 3 años, el 35,1\% (13) 4 años, el 10,8\% (4) 5 años y el 5,4\% (2) 6 años (Fig. 1).

En relación al período de amamantamiento, encontramos que el 21,6\% (8) no se amamantó, el $24,3 \%$ (9) fue amamantado exclusivamente desde el nacimiento hasta los 6 meses, el 40,5\% (15) recibio amamantamiento con alimentación complentaria, biberon e incorroporación de alimentos hasta los 12 meses, el 5,4\% (2) alimentación completa y continuaron amamantantoun grupo hasta los 18 meses y el 8,1\% (3) y el otro grupo hasta los 24 meses (Fig. 2).

En cuanto al período de inicio de la alimentación por formula se encontró que el 24,32\% (9) se alimenta por formula desde el nacimiento, el $64,86 \%$ (24) y dos grupos comenzarón alimentación mixta con biberon uno entre el primero y el sexto mes y el 10,81\% (4) el otro entre el séptimo y el décimo segundo mes.

Al observar el desarrollo de enfermedades alérgicas reportadas por las madres encontramos que el 35,14\% (13) si presentaba y el 64,86\% (24) no. Así mismo, el 35,14\% (13) presentan enfer- medades de otorrinolaringología y el 64,86\% (24) no.

En relación a los hábitos reportados por las madres en la muestra estudiada encontramos que el 8,11\% (3) presenta succión del pulgar y el 91,89\% (34) no. El 100\% (37) de los individuos no reportaron hábito de succión de objetos, ni mastica objetos, así como tampoco se observó onicofagia.

El 21,62\% (8) de los individuos presentan uso de chupete y el 78,38\% (29) no. Así mismo el $5,41 \%$ (2) succiona pañal y el 94,59\% (35) no, el $48,65 \%$ (18) aun toma tetero y el 51,35\% (19) no, el 18,92\% (7) presenta deglución atípica y el $81,08 \%$ (30) no, y por último el 43,24\% (16) de los individuos es respirador bucal y el 56,76\% (21) no.

\section{Análisis Correlacional:}

Se realizó la correlación de las variables período de amamantamiento y período de inicio de formula con biberones con las variables Alergias y Enfermedades otorrinolaringología, y las varia- 
Tabla 1. Correlación de Pearson entre la variable período de amamantamiento y las variables enfermedades alérgicas, de otorrinolaringología y las pertenecientes a hábitos.

\begin{tabular}{|c|c|c|c|}
\hline & \multicolumn{2}{|c|}{$\begin{array}{l}\text { Período de Ama- } \\
\text { mantamiento } \\
\text { mayor a } 6 \text { meses }\end{array}$} & \multirow[t]{3}{*}{$\begin{array}{l}\text { Nivel } \\
\text { Sig. }\end{array}$} \\
\hline & Pearson & $\begin{array}{l}\text { Sig. } \\
\text { Estad. }\end{array}$ & \\
\hline & $(\rho)$ & $(\alpha)$ & \\
\hline Enf. Alérgicas & 0,553 & 0,000 & ** \\
\hline $\begin{array}{l}\text { Enf. Otorrinolaringo- } \\
\text { logía }\end{array}$ & 0,653 & 0,000 & $* *$ \\
\hline Succión Pulgar & 0,318 & 0,055 & NS \\
\hline Uso Chupete & 0,600 & 0,000 & $* *$ \\
\hline Succión de Pañal & 0,114 & 0,500 & NS \\
\hline Uso biberón & 0,418 & 0,010 & * \\
\hline Deglución Atípica & 0,537 & 0,001 & $* *$ \\
\hline Respiración Bucal & 0,708 & 0,000 & $* *$ \\
\hline
\end{tabular}

bles pertenecientes a hábitos: succión de pulgar, pañal y de otros objetos, uso de chupete, mastica objetos, onicofagia, uso de biberón, deglución atípica y respirador bucal.

Se encontraron correlaciones positivas y estadísticamente significativas entre la variable período de amamantamiento y las variables enfermedades alérgicas y otorrinolaringología ( $\rho$ 0,553 - $\alpha$ 0,$000 ; \rho 0,653-\alpha 0,000$ respectivamente) (Tabla 1).

Así mismo se encontraron correlaciones positivas y estadísticamente significativas entre el período de amamantamiento y las variables pertenecientes a hábitos: uso de chupete $(\rho$ 0,600 - $\alpha$ $0,000)$, uso de biberon $(\rho 0,418-\alpha 0,010)$, deglu-
Tabla 2. Correlación de Pearson entre la variable período de inicio de tetero y las variables enfermedades alérgicas, ORL y las pertenecientes a hábitos.

\begin{tabular}{|c|c|c|c|}
\hline \multirow{2}{*}{} & \multicolumn{2}{|c|}{$\begin{array}{c}\text { Período de inicio } \\
\text { de Biberón }\end{array}$} & $\begin{array}{c}\text { Nivel } \\
\text { Sig. }\end{array}$ \\
\cline { 2 - 4 } & Pearson & $\begin{array}{c}\text { Sig. } \\
\text { Estad. }\end{array}$ & \\
\cline { 2 - 4 } & $(\rho)$ & $(\alpha)$ & \\
\hline Enf. Alérgicas & 0,514 & 0,001 & $* *$ \\
\hline Enf. ORL & 0,711 & 0,000 & $* *$ \\
\hline Succión de Pulgar & 0,274 & 0,101 & NS \\
\hline UsoChupete & 0,560 & 0,000 & $* *$ \\
\hline Succión de Pañal & $-0,056$ & 0,742 & NS \\
\hline Uso Biberón & 0,428 & 0,008 & $* *$ \\
\hline Deglución Atípica & 0,485 & 0,002 & $* *$ \\
\hline Respiración Bucal & 0,646 & 0,000 & $* *$ \\
\hline *Sig. 0,05; **Sig. 0,000; NS. No Significativo \\
\hline
\end{tabular}

*Sig. 0,05; ${ }^{* *}$ Sig. 0,000; NS. No Significativo

ción atípica $(\rho, 537-\alpha 0,001)$ y respirador bucal ( $\rho, 708-\alpha 0,000)$. No se encontraron correlaciones con succión de pulgar $(\rho 0,318-\alpha 0,055)$ y succión del pañal ( $0,114-\alpha 0,500)$ (Tabla 1).

Estos resultados podrían indicar que aquellos individuos que fueron amamantados por más de 6 meses presentaron menos enfermedades alérgicas y menos enfermedades de otorrinolaringología, y menos hábitos viciosos bucales: uso de chupón, uso de biberón, deglución atípica y respirador bucal. 
Se encontraron correlaciones positivas y estadísticamente significativas entre el período de inicio de biberón y las variables pertenecientes a hábitos viciosos bucales: uso de chupete $(\rho, 560$ $-\alpha 0,000)$, uso de biberon ( $0,428-\alpha 0,008)$, deglución atípica $(\rho 0,485-\alpha 0,002)$ y respirador bucal ( $\rho, 646-\alpha 0,000)$. No se encontraron correlaciones con succión de pulgar $(\rho 0,274-\alpha$ $0,101)$ ni con succión de pañal $(\rho-0,056-\alpha$ 0,742) (Tabla 2).

En relación a la variable período de inicio de biberon y las variables enfermedades alérgicas y otorrinolaringología se encontraron correlaciones positivas y estadísticamente significativas ( $\rho$ $0,514-\alpha 0,001 ; \rho 0,711-\alpha 0,000$ respectivamente) (Tabla 2).

\section{Discusión}

En los últimos años, el amamantamiento ha sido promovido por organismos internacionales como UNICEF y OMS y en Venezuela por la Comisión de Lactancia Materna Nacional (CONALAMA) como el alimento más completo para los recién nacidos, debido a que este tipo de alimentación favorecerá el correcto desarrollo y crecimiento de los lactantes. La influencia del amamantamiento en el desarrollo del complejo buco-dento-maxilo-facial ha sido pobremente estudiada en nuestro país por muy pocos investigadores, no existiendo de esta manera evidencia científica confiable para realizar las comparaciones debidas entre diferentes grupos de lactantes, y evaluar de esta manera la influencia ${ }^{6}$.

El presente estudio, tuvo como finalidad determinar la influencia del amamantamiento por 6 meses o más en relación con la presencia de hábitos nocivos bucales. Tuvimos limitaciones para realizar el trabajo, debido a que algunas madres se negaban a participar y otras eran lla- madas a la consulta y luego no quisieron continuar con el examen clínico bucal, no se encontró en la literatura revisada estudios previos que senalar este tipo de limitación.

Las madres de este estduio no habían culminado el bachillerato, esta homogeneidad permitió realizar las comparaciones debidas con mayor significancia. Además dado que los niños fueron evaluados en edades entre 3 y 6 años, el estudio muestra diversos factores ambientales, tales como hábitos de succión que también contribuyen con el crecimiento mandibular tal como sucedió en otros estudios realizados en Vene$z_{\text {zuela }}^{7}$, y en la India ${ }^{8}$ quienes analizaron a niños entre estas edades también.

Moimaz A y clos. ${ }^{9}$ en un estudio que se tomaron en cuenta todas las formas de amamantamiento dio como resultado estadístico una asociación negativa significativa con la presencia de hábitos de succión, lo cual difiere del presente estudio en el cual observamos que el período de amamantamiento tiene una correlación positiva significativa con respecto a la presencia de hábitos viciosos bucales al igual que un estudio realizado en la Universidad De Puerto Rico ${ }^{10}$.

Se determinó también en nuestro estudio que la correlación entre el amamantamiento y la succión del pulgar no era significativa por lo cual se encontró la presencia de succión del pulgar en niños amamantados, lo cual coincide con el estudio del autor Moimaz'.

Nuestro estudio no coincide con el estudio realizado por Rodríguez González ${ }^{11}$, donde predominó la succión digital, aunque no tuvieron en cuenta el sexo sino la edad.

Se observa la concordancia de este estudio con el realizado por Claudino y Traebert ${ }^{12}$ sobre la percepción de la calidad de vida, donde de- 
muestran que el hábito más frecuente es el uso de chupetes y biberones.

El principal problema del hábito de succión de chupete consiste en causar dificultades en el período de instauración de la lactancia materna. Según Fonti, el uso de pacificadores causa una interferencia o efecto sobre la duración del período de amamantamiento, lo que no fue observado en nuestro estudio donde el uso del chupete no se le responsabiliza al abandono precoz de la lactancia materna ${ }^{13}$.

Según Barroso Espadero D ${ }^{6}$. Durante un foro pediatrico realizado en el 2013. El deseo de succión está relacionado con la capacidad de supervivencia, ya que le permite al recién nacido alimentarse, desarrollarse y crecer, además de que experimentan una sensación de seguridad derivada de la succión en si misma obteniendo una sensación placentera, reconfortante y proporcionadora de seguridad.

En este estudio el porcentaje de individuos que desarrollaron el hábito nocivo bucal de succión de pulgar fue muy bajo obteniendo así una correlación negativa, ya que mientras mayor sea el período de amamantamiento menor será el hábito de succión.

El amamantamiento exclusivo por 6 meses y mantenerlo con alimentación complemtaria, como lo recomienda la Organización Mundial de la Salud hasta los 2 años y medio tiene mayor influencia en la maduración de las funciones del aparato bucal ${ }^{14}$, en comparación con la alimentación de biberón, esto coincide con los resultados de Munayco ${ }^{15}$, quién encontró que los niños que no han tenido lactancia materna presentaron menores indicadores de crecimiento mandibular, así como Sánchez-Mollins ${ }^{16}$, quien estudió la comparación entre tipos de lactancia y creci- miento dento-buco-maxilo-facial. Otros estudos tambén concluyen que la lactancia materna favorece un correcto desarrollo craneo-facial, en los cuales se encontrando cambios dentales, esqueléticos y estéticos entre los individuos que no recibieron lactancia materna y aquellos que sólo fueron amamantados ${ }^{16 ; 17 ; 18 ; 19 ; 20}$.

Algunos autores asumen que los recién nacidos que son amamantados por un período largo de tiempo tienen menos probabilidades de iniciar el hábito de succión digital 21;22:23:24, lo que concuerda fuertemente con nuestro análisis de estudio.

Saliba $S$ y cols. ${ }^{25}$ al igual que Medeiros E y cols. ${ }^{26}$ coinciden que la prevalencia en la aparición de hábitos de succión no nutritivos no está significativamente asociada con ninguna variable demográfica en sus estudios, mientras que si tuvo relación con la duración del período de lactancia materna.

El porcentaje de individuos que presentó uso de biberón fue casi la mitad de la población estudiada, lo cual nos confirma que la lactancia artificial es un factor etiológico principal en el desarrollo de hábitos viciosos bucales coincidiendo con resultados de otros estudios realizados en Venezuela y España $27: 28$.

En el presente estudio se observó que el inicio de la alimentación por lactancia artificial fue muy temprano, abarcando un $64 \%$ de la población estudiada. Dando como resultado una correlación positiva estadísticamente significativa entre el uso del biberon y la deglución atípica, coincidiendo con autores como Mayoral ${ }^{29}$ quién plantea que la persistencia de la alimentación con formula va unida a la deglución anormal, causando anomalías dentofaciales.

La asociación de la lactancia materna y la lactancia artificial con el desarrollo del hábito de 
chupete coincide en nuestro estudio con los resultados obtenidos por Mendes ${ }^{30}$, el cual encontró relación entre este hábito y la prevalencia de overjet moderado o severo, overbite, mordida abierta anterior y mordida cruzada posterior por uso de chupete.

Al igual que lo observado en nuestro estudio. El amamantamiento constituye para el lactante el intercambio más importante con el mundo exterior. Con esa acción no sólo se alimenta, sino que recibe la sensación de euforia y bienestar al satisfacer aquellos requisitos de seguridad, amor y establecer un contacto físico (táctil, sonoro y bucal) gratificante para él y su madre. Durante los primeros meses de vida, durante el amamantamiento el niño debe presionar con la lengua los senos lactíferos para obtener la leche, pero no necesita esfuerzo alguno para darle forma a la tetilla dentro de su boca cuando se alimenta mediante el biberón. Si el niño succiona y regula el fluido con la lengua, la reacción de estrés normal puede dar inicio a otros hábitos como el de deglución atípica ${ }^{31}$.

Al analizar la correlación entre las variables de período de inicio de biberón y las variables de enfermedades alérgicas se determinó una corre- lación positivamente significativa, la cual concuerda con un estudio realizado en España donde evaluaron a una población de 1.000 niños, la mitad de los cuales habían sido amamantados, donde analizaron la función respiratoria cada dos a cinco años hasta que cumplieron 26 años de edad, se les practicaba un test cutáneo para determinar si presentaban predisposición alérgica; concluyendo que mientras más temprana sea el inicio de la lactancia artificial mayor será la posibilidad de presencia de enfermedades alérgicas $^{31}$.

\section{Conclusión}

En este estudio se observó una alta significancia estadística al relacionar los hábitos viciosos bucales con el período de amamantamiento, se destaca que en cuanto a succión digital y uso de pañal la relación no fue relevante.

\section{Recomendación}

Estudios sobre el tema en poblaciones más grandes deben realizarse para precisar con más detalles las variables estudiadas.

\section{Bibliografía}

1. López Y, Arias M, Zelenenko O. Lactancia materna en la prevención de anomalias dentomaxilofaciales. Rev. Cubana Ortop. 1999; 14(1): p. 32-38.

2. Blanco L, Guerra M, Mujica C. Relación entre el amamantamiento, el tipo de perfil, oclusión y hábitos viciosos en preescolares. Arch. Venez de Pueri Pediatr. 1999; 62: p. 138-143.

3. Godoy D, Haller W, Casamayou M. Prevención de las disgnacias desde el nacimiento. ¿Es posible? [Online]. [cited 2013 julio. Available from: http:/ / www.odon.edu.uy.

4. Santiso A, Santiso Y, Cidre F, Gómez I. Evaluación del tiempo de lactancia materna como factor de riesgo en alteraciones de la oclusión dentaria temporal. Mediciego. 2011; 17(1).

5. Lima Álvarez M, Romero E, Pérez R. Hábitos bucales deformantes en un consultorio del médico de la familia en Camaguey, Cuba. [Online].; 2004 [cited 2013 julio. Available from: www.amc.sld.cu/amc/2004/v8n4/928.htm.

6. Barroso Espadero D. Foro Pediátrico. [Online].; 2013 [cited 2014 Junio 8 [Foro Pediátrico]. Available from: www.spapex. es. 
7. Guerra M, Blanco L, Mujica C. Relación entre período de amamantamiento y desarrollo maxilar en niños indígenas pemones venezolanos. Bol Asoc Argent Odontol Niños. 2006 marzo; 35(1): p. 11-14.

8. Ganesh M, Tandon S, Sajida B. Prolonged feeding practice and its effects on developing dentition. J. Indian Soc Pedod Prev Dent. 2005; 23: p. 141-5.

9. Moimaz A, Zina L, Saliba N, Saliba O. Association between breast-feeding practices and sucking habits: a cross-sectional estudy of children in their first year of life. Journal of Indian Society of Pedodontics and Preventive Dentistry. 2008; 26(3): p. 102-106.

10. López del Valle L, Feliciano N, Machuca M. Associations between a history of breast feeding, malocclusion and parafuntional habits in Puerto Rican children. Puerto Rico Health Sciences Journal. 2006 Marzo; 25(1)

11. Rodríguez González A, Martínez Brito I. Influencia de la lactancia materna en el micrognatismo transversal y los hábitos bucales deformantes. Rev Med Electron. 2011; 33(1).

12. Claudino, Traebert. Malocclusion, dental a esthetic self-perception and quality of life in a 18 to 21 year-old population: a cross section study. BMC Oral Health. 2013 Jan; 13(3).

13. Fonti M, Canadian Paediatric Society. Recommendations for the use of pacifiers. Paediatr. Child. Health. 2003; 8(8): p. 515-19.

14. Santos D, Véliz O. Influencia de la lactancia materna sobre el arco dentario y los maxilares en niños de cinco años de edad. Revista Actualidad profesional e industrial del sector dental. 2007 Abril; 98.

15. Munayco A, Piedra R, Cortez M. Lactancia materna asociada a crecimiento mandibular en niños de 3 años de edad. Revista Kiru. 2005; II(1): p. 4-10.

16. Sánchez-Molins M. Estudio comparativo del crecimiento cráneo facialsegún el tipo de lactancia recibida. Monografías Clínicas en Ortodoncia. 2007 Ene-Feb; 25(1): p. 9-16.

17. Rondón R, Zambrano G, Guerra M. Relación de la lactancia materna y el desarrollo dento-buco-maxilo-facial. Revista Latinoamericana de Ortodoncia y Odontopediatría. 2012.

18. Benitez L, Calvo L, Quirós O, Maza P, Jurisic D, Alcedo C. Estudio de la Lactancia materna como factor determinante para prevenir las anomalías dentomaxilofaciales. Rev. Latinoamericana de Ortodoncia y Odontopediatría. 2009.

19. Gutiérrez H. Crecimiento Maxilomandibular y maloclusión en escolares de 14 a 16 años de edad y su relación con la lactancia en la provincia de Andahuaylas. 2004. Universidad de San Martín de Porres.

20. Warren J, Bishara S. Duration of nutritive and nonnutritive sucking behaviors and their effects on the dental arches in the primary dentition. Am J Orthod Dentofacial Orthop. 2002; 121: p. 347-56.

21. Najat MAF, Fouad S. S, Cert P. Sucking habits in Saudi children: prevalence, contributing factors and effects on the primary dentition. Pediatric Dentistry. 1997; 19(1): p. 28-33.

22. Saanum M, Bjorn O, Larsson E. The influence of dietary habits, breastfeeding and sucking habits on the development of the occlusion in the primary dentition. 2002. A clinical study on Mexican children 2000-2002. University Oslo.

23. Narbutyte I, Narbutyte A, Linkeviciene L. Relationship between breastfeeding, bottle-feeding and development of malocclusion. Stomatologija, Baltic Dental and Maxillofacial Journal. 2013; 15: p. 67-72.

24. Batista S, Oliveira T, De Lima F, Castro M, Periara A. Assoaciation of breastfeeding pacifier use, breathing pattern and malocclusions in preschoolers. Dental Press J Orthod. 2003 ene-feb; 18(1)

25. Saliba S, Saliba O, Lolly LF, Saliba C, Isper A, Saliba N. A longitudinal study of the association between breast-feeding and harmful oral habits. Pediatr. Dent. 2012; 34: p. 117-21.

26. Medeiros E, Rodrigues M. A importancia da amamentacao natural para o desenvolvimiento do sistema estomatognático do bebe. Rev. Cons. Reg Odontol. Pernambuco. 2001 julio/Dez; 4(2): p. 79-83

27. Blanco-Cedres L, Guerra M, Rodríguez S. Lactancia materna en la prevención de hábitos orales viciosos de succión y deglución. Acta Odontológica Venezolana. 2007 Noviembre; 45(1)

28. Planells P, Martin S, Echaniz R. Hábitos de succión digital y chupete en el paciente. Odontología Pediátrica. 2003; 12(2): p. $85-90$

29. Mayoral J, Mayoral G. Ortodoncia: Principios fundamentales y práctica. 3rd ed. Barcelona : Labor; 1969. 
30. Mendes R, Adriana C. Associacao Entre Tipos e Tempo de Aleitamento, Hábitos de Succao Nao-Nutritivos e Maloclusoes em Criancas de 3 a 5 Anos na Cidade de Joao Pessoa-PB. [Online].; 2005 [cited 2013 julio. Available from: http/ / redalyc. uaemex.mx/ redalyc/src/inicio / ArtPdfred.jsp?iCve $=6375011$.

31. CASOS CLÍNICOS INTERACTIVOS EN ALERGÍA PEDIÁTRICA. [Online].; 2011 [cited 2014 Junio 30 [Congreso de la Sociedad Española de Alergología e Inmunología Clínica]. Available from: http: / / www.aeped.es / sites/default/files / AEP_Ponencias_2011.pdf.

Recibido 03/08/2014

Aceptado 19/12/2014

Correspondencia.dickmarlozada@hotmail.com 